
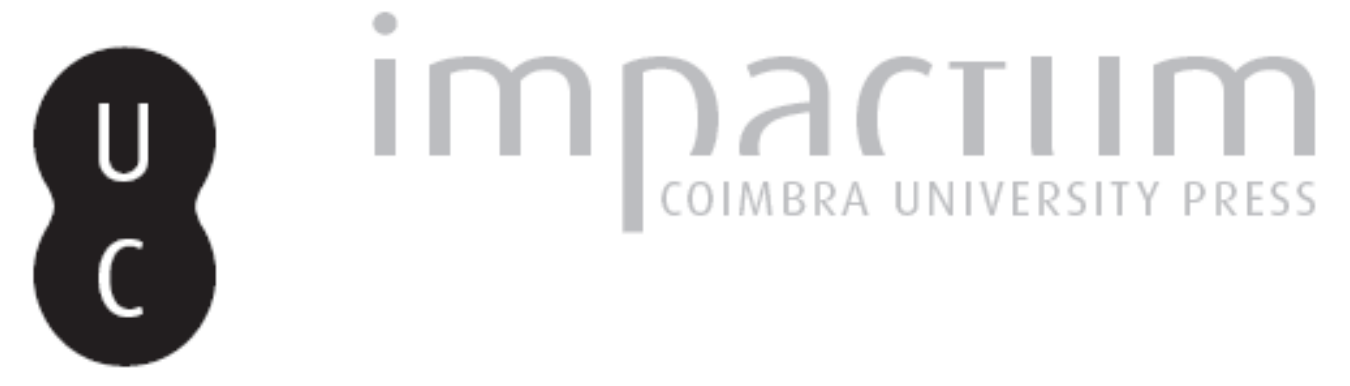

\title{
Planeamento alimentar na redução das vulnerabilidades e melhoria da capacidade de resposta municipal em emergência civil
}

\author{
Autor(es): $\quad$ Lameiras, Jorge
}

Publicado por: Associação Portuguesa de Riscos, Prevenção e Segurança

URL persistente:

URI:http://hdl.handle.net/10316.2/36113

DOI:

DOI:http://dx.doi.org/10.14195/1647-7723_17_24

Accessed : $\quad$ 26-Apr-2023 10:27:31

A navegação consulta e descarregamento dos títulos inseridos nas Bibliotecas Digitais UC Digitalis, UC Pombalina e UC Impactum, pressupõem a aceitação plena e sem reservas dos Termos e Condições de Uso destas Bibliotecas Digitais, disponíveis em https://digitalis.uc.pt/pt-pt/termos.

Conforme exposto nos referidos Termos e Condições de Uso, o descarregamento de títulos de acesso restrito requer uma licença válida de autorização devendo o utilizador aceder ao(s) documento(s) a partir de um endereço de IP da instituição detentora da supramencionada licença.

Ao utilizador é apenas permitido o descarregamento para uso pessoal, pelo que o emprego do(s) título(s) descarregado(s) para outro fim, designadamente comercial, carece de autorização do respetivo autor ou editor da obra.

Na medida em que todas as obras da UC Digitalis se encontram protegidas pelo Código do Direito de Autor e Direitos Conexos e demais legislação aplicável, toda a cópia, parcial ou total, deste documento, nos casos em que é legalmente admitida, deverá conter ou fazer-se acompanhar por este aviso.

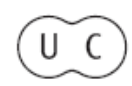




\section{territorium}

Riscos, Sociedade(s) e Segurança

Revista da Associação Portuguesa de Riscos, Prevenção e Segurança 2010 


\title{
PLANEAMENTO ALIMENTAR NA REDUÇÃO DAS VULNERABILIDADES E MELHORIA DA CAPACIDADE DE RESPOSTA MUNICIPAL EM EMERGÊNCIA CIVIL ${ }^{1 *}$
}

\author{
Jorge Lameiras \\ Instituto Piaget / Viseu \\ jlameiras@viseu.ipiaget.org
}

A existência de mecanismos de acompanhamento da sociedade portuguesa contemporânea em matérias como a estrutura demográfica, o poder económico das famílias ou a vigilância epidemiológica (quer em termos dos níveis actuais de saúde, quer em termos das preocupações dominantes em matéria de doença e de segurança alimentar), incluindo o conhecimento sobre o modo como todos aqueles aspectos têm vindo a evoluir e se projectam no futuro, significa a disponibilidade de informação que pode ser mobilizada para elaborar um quadro social e de saúde, sobre o qual os sistemas de apoio à população em situação de catástrofe poderão criar e experimentar planos de prevenção, preparação e resposta.

A alimentação praticada por cada indivíduo e, de uma forma mais ampla, por cada comunidade ou grupo dentro de uma mesma comunidade, é determinada por numerosos factores de ordem biológica, do ambiente natural, social, económica, cultural e política. Deste modo, a diferentes grupos sociais correspondem diferentes padrões alimentares, o que condiciona a expressão de doenças e a mortalidade associada a esses grupos sociais (ROBERTSON, 2004). Condições como a pobreza, ou pelo menos a situação de dificuldade económica marcada e continuada, sobretudo em associação com défices de informação (socioculturais) e em infra-estruturas sanitárias, é um factor socioeconómico determinante para as doenças infecciosas e parasitárias. Essa influência também se verifica no aumento das doenças por contaminação microbiológica dos alimentos (ROBERTSON, 2004). Porém, aquela condição também é cada vez mais valorizada como factor de morbilidade e mortalidade por doenças insidiosas do foro vascular, metabólico, digestivo e oncológico (МАRMOT, 2005). Face a isto, o crescente desenvolvimento (político e socioeconómico, educacional e cultural) com melhoria das condições de saúde pode ser apontado como factor de redução da vulnerabilidade das comunidades humanas face aos eventos catastróficos.
As situações de catástrofe ao provocarem rupturas no quotidiano dos indivíduos e das comunidades podem gerar dificuldades de acesso à alimentação, seja na quantidade ou diversidade de alimentos disponíveis, seja na forma e tempo das refeições. Esta condição pode ser ainda agravada pela criação de novas necessidades que surgem como consequência clínica de lesões diversas ou de problemas como a perda ou ruptura do fornecimento de medicamentos a doentes crónicos (por exemplo diabéticos e hipertensos).

Aanálise de informação disponível sobre comunidades sujeitas a catástrofes naturais, em países considerados como tendo condições de vida gerais favoráveis à saúde, serve para revelar grupos de risco nutricional em termos de consumos abaixo das recomendações (MAGkos et al, 2004; Callaghan et al, 2007; Leitmann, 2007).

Aspectos biológicos como o aumento das necessidades por stress fisiológico, aspectos culturais relacionados com a protecção da criança (prioridades familiares), e aspectos sociais como a perda de rendimento económico são alguns factores de relevo para a instalação de consumos alimentares deficitários em adultos (MAGKos et al, 2004); doenças crónicas, exposição a condições meteorológicas adversas após a catástrofe e dificuldades na preparação das refeições são exemplos de factores adicionais identificados na contribuição para a degradação do estado nutricional na população idosa (Young et al, 2004).

0 interesse do planeamento no domínio alimentar coloca-se em vários níveis de organização: nacional, regional / distrital e local / municipal. Também se pode considerar o nível transnacional, decorrente da integração do Estado português em organizações políticas internacionais, sob cuja égide funcionam diversos sistemas de apoio mútuo.

A União Europeia produziu já um quadro de referência organizacional onde a complementaridade dos princípios de subsidariedade de governação, e de coordenação central, surge enfatizada como meio para garantir uma maior eficiência no processo de aquisição

\footnotetext{
$1^{*} \mathrm{O}$ texto desta nota corresponde à comunicação apresentada ao V Encontro Nacional e I Congresso Internacional de Riscos e foi submetido para revisão em 20-06-2009, tendo sido aceite para publicação em 27-01-2010.

Esta nota é parte integrante da Revista Territorium, n. ${ }^{\circ} 17,2010$, ๔ Riscos, ISBN: 0872- 8941.
} 
do conhecimento sobre as comunidades locais e uma maior eficácia do apoio a essas mesmas comunidades em situações de emergência (UE-CR, 2005; UE-CR, 2006).

As soluções de proximidade são reconhecidas como fundamentais para o apoio imediato à população em situação de catástrofe, independentemente de apoios externos à comunidade, de maior capacidade ou mais diferenciados (UE-CR, 2006), acções que se enquadram do ponto de vista organizacional no conceito de Protecção Civil (UE-CE, 2005). A existência de reservas, a disponibilidade de meios de apoio mobilizáveis e o nível de prontidão dos agentes operacionais são fundamentais para a capacidade de resposta efectiva. A análise de eventos que marcaram a história recente do "mundo ocidental", o "retorno da experiência" como the chamam Guilhou \& LAGADEC (2004), mostra que nas primeiras horas sempre, e mesmo nos primeiros dias após o evento, a resposta tem origem nas entidades locais, com as pessoas e os meios que tenham resistido ao evento. A preparação deve incluir as acções que assegurem um conjunto de reservas de água e alimentos, bem como de fontes energéticas e sistemas de eliminação de resíduos (McSwaln Jr, 2006). Temos, assim, a exigência de um trabalho centrado quer na formação dos agentes com responsabilidade de intervenção, quer na educação da população para a autonomia familiar.

O sistema nacional de Protecção Civil, conforme estruturado ao abrigo da respectiva Lei de Bases (Lei $n^{\circ} 27 / 2006$, de 03/07), estabelece a responsabilidade das autarquias em matéria de direcção e execução da política de Protecção Civil no âmbito territorial dos municípios. Os objectivos e domínios de actuação da Protecção Civil municipal estão perfeitamente definidos na legislação em vigor (Lei $n^{\circ} 65 / 2007$, de 12/11), que prevê também a elaboração e actualização de planos municipais de emergência.

Há um desafio global que se impõe em matéria de resposta alimentar à população em situação de catástrofe: identificar níveis de susceptibilidade e factores de vulnerabilidade, preparar os meios e os procedimentos de acção para quebrar o ciclo da catástrofe. Este não é sequer um desafio novo. Aspectos como a preparação dos intervenientes na Protecção Civil, o aperfeiçoamento dos meios e métodos de previsão, das técnicas e métodos de intervenção e de manutenção imediata após a ocorrência de situações de emergência (nomeadamente no plano logístico), e a informação, formação e sensibilização dos cidadãos foram já realçados (UE-CE, 1999). O investimento na prevenção e preparação parece ser fundamental para contrariar disfuncionalidades em situação de crise (DE GOYET, 1999).

O modo como é planeada e gerida a organização do espaço comunitário influencia a qualidade de vida da população, em aspectos como o acesso a bens e serviços (VLAHov et al., 2007) e a capacidade de resposta logística do sector alimentar em situação decorrente de uma catástrofe (MATHEW \& MCDONALD, 2006).

A preocupação relativa à preparação dos meios de apoio é enfatizada em documento orientador europeu (UE-CR, 2006), face a grupos com características socio-económicas e de saúde especiais, como se nota na relevância dada “...à população infantil e juvenil e a outros sectores especialmente vulneráveis em caso de emergência, como pessoas idosas e de mobilidade reduzida". As dificuldades de mobilidade e recursos económicos escassos, limitadores face aos custos de deslocação e estadia fora da residência, contribuem para dificultar uma decisão autónoma de retirada das zonas afectadas (Brodie et al, 2006).

Referenciais de planeamento alertam para a conveniência de ter em consideração elementos tais como o número previsto de pessoas afectadas e as características demográficas, a estrutura social e política da população, as condições de segurança e operacionais, as políticas existentes em matéria de nutrição, os grupos de risco, as capacidades e os recursos locais, as infraestruturas locais e as instalações e serviços existentes, e as possíveis implicações e impacto ambiental a longo prazo que podem ter as intervenções propostas (SPHERE, 2004). A disponibilidade de meios físicos e fontes energéticas, bem como de condições de conservação de alimentos são tópicos fundamentais no planeamento alimentar para situações de catástrofe (WHO, 2000).

A disponibilidade de água e alimentos não é uma preocupação apenas dirigida à população civil afectada. A implementação de sistemas de apoio alimentar à população civil afectada em situação de emergência, bem como aos agentes operacionais responsáveis pelas operações de socorro e contenção, requer um planeamento logístico rigoroso que antecipe necessidades operacionais em matéria de abastecimento alimentar de qualidade nutricional e nível de segurança irrepreensível, e que evite a exposição a contaminantes de natureza toxicológica, microbiológica ou parasitária que possam afectar a própria operacionalidade do pessoal envolvido nas operações de socorro.

Apesar da consciência de que na alimentação não existe o risco zero, isto é, não é possível organizar uma cadeia alimentar isenta de riscos (ROBERTSON, 2004), as preocupações no sentido de assegurar um abastecimento alimentar quotidiano de boa qualidade nutricional e seguro e a informação e educação alimentar dos consumidores são pontos-chave das políticas internacionais em matéria de política alimentar e nutricional (WHO, 2008) e fazem parte integrante de orientações operacionais em matéria de organização de sistemas de abastecimento alimentar (MOUREY, 2008) e preparação de refeições em campo (EMA, 2003).

As preocupações com a alimentação no quadro legislativo da União Europeia podem também ser enquadradas no campo da segurança das infra-estruturas 
críticas em matéria de bioterrorismo. Esta ideia pode ser inserida na avaliação de riscos, na elaboração de normas de segurança no domínio da protecção de pessoas e daquelas infra-estruturas e na segurança das cadeias de abastecimento, aspectos realçados por normativo europeu (UE-CUE, 2007).

$\mathrm{O}$ trabalho de planeamento padece, às vezes, de uma falta de reconhecimento público notada na dificuldade de mobilização de meios adequados e suficientes (com todas as limitações que decorrem de um certo nível de imprevisibilidade dos eventos) antes da tomada de consciência pública da existência de um risco e antes do desencadeamento de uma crise (GuILHou \& LEGADEC, 2004). Por outro lado, a disponibilidade de restaurantes e de entidades com sistemas de alimentação colectiva na generalidade do território nacional, bem como de outras entidades que realizam apoio social, podem criar uma sensação de segurança em matéria de abastecimento alimentar de emergência. Deve ser ponderado até que ponto não se gera uma noção de infalibilidade do sistema que mascare défices de organização potencialmente cindinogénicos, como refere KERVERN (1995).

Como referem Guilhou \& Lagadec (2004), “a crise não pode ser abordada, prevenida, resolvida através de simples medidas técnicas". Porém, talvez seja importante que haja um ponto de partida, de informação cientificamente validada e capacidade técnica instalada para que haja uma referência sobre o cuidado a dispensar (neste caso, em termos alimentares e nutricionais) e sobre as exigências logísticas que permitem disponibilizar o apoio alimentar mais adequado para que, a partir daqui, seja possível adaptar os métodos e os meios para garantir a autonomia do sistema numa situação de ambiente instável. Os planos não valem apenas pelo aspecto técnico, até porque numa situação de crise as rupturas sociais, a falta de informação e as perdas de meios podem exigir, segundo GUILHOU \& LAGADEC (2004), uma "inteligência criativa" capaz de apreender a situação e estabelecer lógicas de acção que permitam à comunidade iniciar a saída da crise. Os planos também valem pelo estímulo ao desenvolvimento de uma aptidão colectiva para a organização, ou seja por uma cultura de segurança e responsabilidade a que apelam, tanto nas organizações que têm uma missão no domínio da gestão da emergência e dela têm de prestar contas à população, como nos cidadãos pela visibilidade mediática que adquirem e pelas preocupações que geram.

Tomando como ponto de partida algumas preocupações sobre compilação e análise crítica de informação (WHO, 1995; WHO, 2000; WFP, 2005; MOUREY, 2008), com as devidas adaptações à nossa realidade, uma intervenção no domínio da alimentação deve envolver uma multiplicidade de frentes para controlo sobre os pontos críticos em matéria de suficiência, de adequação nutricional e cultural, de qualidade e de segurança, nomeadamente através de:
1. Estudo de factores demográficos e da situação socioeconómica da população com identificação dos grupos mais vulneráveis;

2. Avaliação da capacidade instalada de resposta operacional na área alimentar (planos alimentares, reservas alimentares estratégicas - físicas e/ ou funcionais -, estruturas e equipamento de restauração, pessoal competente mobilizável);

3. Análise de riscos no plano da qualidade e segurança alimentar (microbiológica, parasitológica e toxicológica - incluindo a avaliação de fragilidades em matéria de bioterrorismo) inclusive para garantir que o apoio não seja portador de mais riscos e se torne um foco de novos eventos que se adicionem aos já instalados;

4. Planeamento e gestão de sistemas de apoio alimentar de emergência, que permita assegurar a autonomia do sistema pelo menos nas primeiras 48-72 horas, e prestar apoio necessário a vítimas, pessoal de serviços e pessoal projectado no teatro de operações, e que inclua a implementação de procedimentos de minimização dos riscos mencionados;

5. Formação de pessoal (municipal e dos agentes de Protecção Civil) com funções que envolvam gestão da alimentação;

6. Educação da população na perspectiva da autonomia e segurança alimentar.

Face à inevitabilidade dos eventos de catástrofe, a alimentação enquanto aspecto básico de sobrevivência e saúde deve ser fortemente valorizada no âmbito do planeamento civil de emergência, com relevo para o nível municipal onde as pressões de resposta à população e aos agentes operacionais de Protecção Civil se fazem notar de imediato.

A valorização das questões em torno da alimentação no âmbito do planeamento civil de emergência no nível local / municipal, pode constituir um importante investimento no sentido de fortalecer a capacidade de resposta e a resiliência das comunidades locais. A disponibilidade de alimentos em quantidade suficiente, de boa qualidade nutricional, segura e adequada às necessidades fisiopatológicas e culturais e à disponibilidade de meios de conservação e preparação, requer um conhecimento do terreno e uma capacidade de projecção de meios que não deve ficar apenas dependente do improviso. Simultaneamente, o desenvolvimento de uma cultura de segurança colectiva em que todos os cidadãos percebam o valor do seu contributo para a redução de vulnerabilidades e para a capacidade de resposta às adversidades partilhadas pela comunidade exige uma aposta fortíssima na informação pública e na educação. 


\section{Referências bibliográficas}

BRodie, Mollyann et al. (2006) - "Experiences of Hurricane Katrina Evacuees in Houston Shelters: Implications for the Future Planning". American Journal of Public Health, 96 (5), p.1402-1408.

Callaghan, William et al. (2007) - "Health Concerns of Women and Infants in Times of Natural Disasters: Lessons Learned from Hurricane Katrina". Maternal Child Health Journal, 11, p. 307-311.

De GoYet, Claude de Ville (1999) - Stop Propagating Disaster Myths. DERA Newsletter. pp. 1-3.

Emergency Management Australia (2003) - Emergency Catering. EMA, Dickson. 115 p.

Guilhou, Xavier \& Lagadec, Patrick (2004) - O Fim do Risco Zero. Lisboa, Ed. Piaget.

Leitmann, Josef (2007) - "Cities and calamities: Learning from Post-Disaster Response in Indonesia". Journal of Urban Health: Bulletin of the New York Academy of Medicine, 84 (1), p. i144-i153.

MAGKos, Faidon et al. (2004) - "Identifying Nutritionally Vulnerable Groups in case of Emergencies: Experience from the Athens 1999 Earthquake". International Journal of Food Sciences and Nutrition, 55 (7), p. 527-536.

MARMOT, Michael (2005) - "Social Determinants of Health Inequalities". The Lancet, 365, p. 1099-1104.

Mathew, Richard \& Mcdonald, Bryan (2006) - "Cities Under Siege - Urban Planning and the Threat of Infeccious Diseases". Journal of the American Planning Association, 72 (1), p. 109-117.

McswaIN Jr., Norman (2006) - “Disaster preparedness perspective from 90.05.32w, 29.57.18n". Critical Care, 10, p.108 (disponível online http://ccforum. com/content/10/1/108)

MOUREY, A. (2008) - Nutrition Manual for Humanitarian Action. ICRC, Genebra, 700 p.

KERVERN, Georges-Yves (1995) - Elementos Fundamentais das Ciências Cindínicas. Lisboa, Ed. Piaget.

Robertson et al (ed.s). 2004. Food and Health in Europe: A New Basis for Action. WHO Regional Office for Europe, Copenhagen. 405 p.
The Sphere Project (2004) - Humanitarian Charter and Minimum Standards in Disaster Response. The Sphere Project, Geneva. 344 p.

VLAHOV, David et al. (2007) - "Urban as a Determinant of Health". Journal of Urban Health: Bulletin of the New York Academy of Medicine, 84 (1), p. i16-i26.

World Food Programme (2005) - Emergency Food Security Assessment Handbook. WFP, Roma.

World Health Organization (1995) - Field Guide on Rapid Nutritional Assessment in Emergencies. WHO Regional Office for the Eastern Mediterranean, Alexandria, $64 \mathrm{p}$.

World Health Organization (2000) - The Management of Nutrition in Major Emergencies. Geneva, WHO, 236 p.

World Health Organization (2008) - Who European Action Plan for Food and Nutrition Policy 2007-2012. Copenhagen, WHO, $28 \mathrm{p}$.

Young, Helen et al. (2004) - "Public Nutrition in Complex Emergencies”. The Lancet, 365, p. 1899-1909.

Outras fontes:

Lei $n^{\circ}$ 27/2006, de 03/07 - Lei de Bases da Protecção Civil. D.R. $\mathrm{n}^{\circ} 126$, Série I, p. 4696-4706.

Lei $n^{\circ}$ 65/2007, de 12/11 - Enquadramento institucional e operacional da Protecção Civil no âmbito municipal.

União Europeia, Conselho da União Europeia - Decisão (1999/847/CE), de 09/12/1999.

União Europeia - Parecer do Comité das Regiões 2005. (2005/C43/10), de 18/02/2005.

União Europeia, Comissão Europeia. COM(2005) 137 final, de 20/04/2005, «Melhorar o Mecanismo Comunitário de Protecção Civil».

União Europeia - Parecer do Comité das Regiões. Jornal Oficial da União Europeia, n C206, de 29/08/2006, pp. C206/9- C206/16, «As catástrofes naturais (incêndios, inundações e seca)».

União Europeia, Conselho da União Europeia (2007). 2007/124/CE, Euratom. «Prevenção, preparação e gestão das consequências em matéria de terrorismo e outros riscos relacionados com a segurança». 